\title{
RETRACTABLE CARBON FIBRE TARGETS FOR MEASURING BEAM PROFILES AT THE SLC COLLISION POINT *
}

\author{
G. BOWDEN, D. BURKE and C. FIELD \\ Stanford Linear Accelerator Center, Stanford University, Stanford, CA 94309, USA \\ W. KOSKA \\ University of Michigan, Ann Arbor, MI 48109, USA
}

Received 23 January 1989

Retractable targets of carbon fibres with nominal diameters of 30,7 and $4.5 \mu \mathrm{m}$ have been in use at the collision point of the SLC (the SLAC Linear Collider). The target mechanism is compatible with the Mark II detector now in place at the collision point. Beam profiles are measured in horizontal and vertical axes by using magnetic dipoles to scan the beams across the fibres. Two signal detection mechanisms are available. Electron emission from the fibres is measured with the help of charge-sensitive amplifiers. Bremsstrahlung from the fibres is detected by downstream counters. Examples of measurements are given, and limits on the use of the techniques are discussed.

\section{Collision point beam profiles}

One of the many challenges at linear colliders is to find methods to measure beam spot dimensions at the collision point, both for beam tuning and operation in colliding mode. At the SLC, the collider presently being commissioned at the Stanford Linear Accelerator Center, the beams have been probed by targets consisting of fine carbon fibres [1]. The beam transverse rms widths, during tuning, vary over the approximate range of 3 to $50 \mu \mathrm{m}$, with bunch intensities between $2 \times 10^{9}$ and $1.5 \times 10^{10}$.

The device described in ref. [1] has already shown that such beam profiles can be measured, with micron accuracy, by mechanically scanning a fibre across the beam while measuring the surface electron emission from the carbon. Before the best focus and highest intensities were available, however, it was necessary to install the Mark II [2] detector in preparation for particle physics data taking. The device to be described was developed to be compatible with the Mark II at the collision point.

\section{Description of target}

The design answered several requirements. It had to: fit inside the inner $38 \mathrm{~cm}$ diameter of the Mark II drift

* Work supported by United States Department of Energy, contracts DE-AC03-76SF00515 and DE-AC02-ER01112. chamber; introduce a minimum of scattering material for azimuthal angles greater than $20^{\circ}$ or less than $7^{\circ}-$ i.e. in front of the main drift chamber and the drift tubes of the small angle detector; and stay outside the synchrotron radiation stay-clear, a $2.5 \mathrm{~cm}$ radius about the nominal beam axis. (Note that no vertex detector was to be installed inside the main drift chamber during this period, and so the outer radial constraint was the main drift chamber itself.)

In order to function at all, the SLC has been provided with a means of positioning the beams at the collision point with a resolution better than a micron [3]. This is done with a combination of iron core dipoles to provide a wide steering range, and air core magnets which afford fast and repeatable adjustments within their limited ranges. It was natural to make use of this ability. Consequently the only mechanical action needed in the new device is insertion of the fibres to, and retraction from, the beam axis.

The absolute positioning tolerances are not particularly severe. In order to remain within the range of good beam optics, it was necessary that, on installation, the fibres could be aligned on the nominal beam line within approximately $750 \mu \mathrm{m}$. Short-term repeatability is more rigorous: if the fibres were to be reinserted on to the beam line within a few hours of being retracted, they should return to their "in" position within $25 \mu \mathrm{m}$. There should be no vibration or other movement of the fibres greater than $1 \mu \mathrm{m}$ when on the beam line. This would be sufficient for measuring beam profiles and 


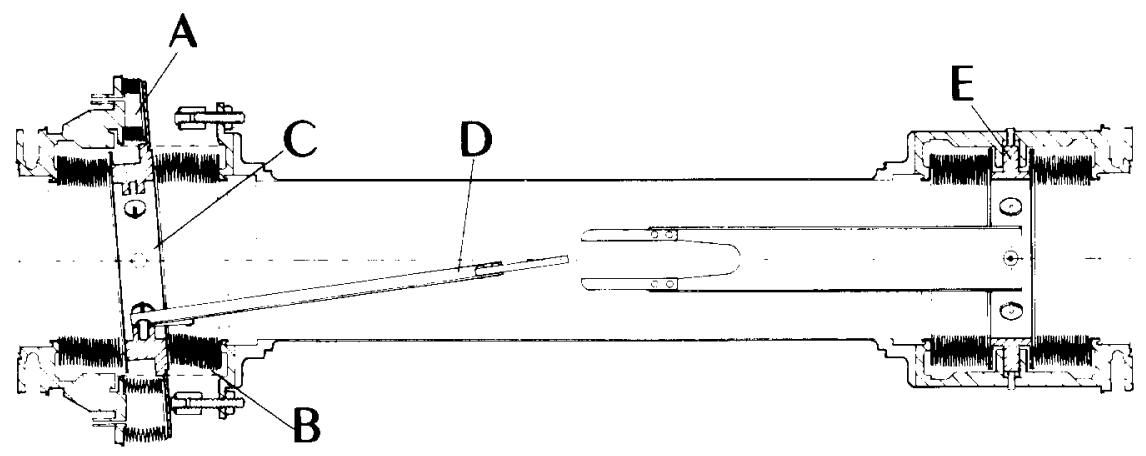

$10 \mathrm{CM}$.

Fig. 1. Layout of the retractable target device. The drawing shows the central beam pipe sectioned longitudinally in the mid-plane, with one cantilever moved to the "in" position; A: pneumatic actuating bellows, B: vacuum bellows, C: rocking ring, D: fibre-supporting cantilever, E: pivot.

would allow a pair of orthogonal fibres to act as locators for bringing the two beams into collision.

A drawing of the device, fig. 1, shows that it is integral with the central part of the beam vacuum pipe. On either end of the $47 \mathrm{~cm}$ pipe is an actuation mechanism. The mechanism was designed to avoid any sliding or rolling contact internal to the $\approx 10^{-9}$ Torr vacuum, thereby avoiding difficulties with bearing adhesion. For the same reason, mechanical contacts for limiting the range of movement were kept outside the vacuum.

As can be seen in the figure, the central piece of the movement is a stainless steel ring, forming part of the vacuum pipe. Cylindrical pins are attached to this diametrically, as trunnions, and the ring is supported by these so as to allow it to pivot. The bearings for the trunnions are held in brackets which bridge between upstream and downstream parts of the nonmoving sections of the beam pipe. This structural material is stainless steel, and the vacuum envelope is completed by welding a pair of stainless steel bellows between the ring and the upstream and the downstream rigid sections. When the ring is rocked, the bellows are forced into a combination of of set and bend distortions. The structure is completed by spacing the two mechanisms by a $25 \mathrm{~cm}$ long, $6.8 \mathrm{~cm}$ diameter, $0.8 \mathrm{~mm}$ thick aluminum pipe, satisfying the need to minimise scattering. The stainless steel to aluminum transition is made of stock explosion-bonded material. Other dimensions of interest are accessible on the drawing, which also indicates that this complete target section of the beam pipe is coupled to the rest of the vacuum system by means of standard high vacuum flanges.

Welded into the rocking ring are four, ceramic insulated, high vacuum, electrical feedthroughs. On the outside, these are formed into standard SMA sockets, and connect with coaxial signal cables. On the inside, they provide a connecting pin for electronic signals from the target fibres. Also brazed to the inside of the ring are small pedestals to which the fibre holders are to be attached. The pedestals are positioned at $90^{\circ}$ from the pivot axis, where the motion is maximum.

The actuating mechanism is powered by air pressure. On the outside of each rocking assembly are mounted two expansion chambers formed of $26 \mathrm{~mm}$ diameter stainless steel bellows, closed at both ends except for a small diameter gas pipe. The chambers are supported on the fixed part of the beam pipe, and push against a bracket forming an extension of the rocking ring. Only one chamber is pressurized at a time, and it rocks the ring in one direction only.

The angular range is determined by screws which are adjusted to limit the movement of these brackets. The shaft of each screw is insulated from the rest of the assembly, and it is used to give an electrical indication when the rocking mechanism is against the stop. After alignment, and with full vacuum load, a pressure of 80 psi is adequate to provide the full range of rotation in each direction.

The rotational axes on the two ends are at right angles to each other. This provides fibre targets with both vertical and horizontal orientation.

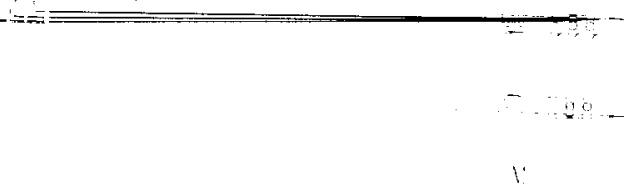

Fig. 2. Cantilever design. The fibres, F, are supported between Macor blades, M. Signals are extracted through thin coaxial "cables", C. 
A drawing of the cantilever fibre mount is shown in fig. 2. The length of the arm is chosen so that the fibres supported from both north and south sides can be on the beam axis at once without touching - there is 2.5 $\mathrm{mm}$ between them in this case. They are attached to the pedestal on the rocking ring by a screw with a low profile head, and aligned by two pins. At the attachment end, the units have a $4^{\circ}$ wedge, which serves to position them at this angle relative to the beam axis when the ring is in the neutral position. The rocking motion is constrained so that, in the "out" position, the cantilever is rotated parallel to the beam axis, and outside a $26 \mathrm{~mm}$ radius. This means that the pneumatic pressure must be maintained to hold the target out when it is not in use. The "in" position is determined by the alignment of the middle fibre with the axis of the beam pipe. Thus the unit rotates approximately $4^{\circ}$ on either side of the neutral position, but in fact is held at all times in either extreme position.

Note that the alignment was referred to tooling balls on the outside of the stainless steel structure, so that the position on the beam line could be determined without access to the fibre targets themselves. Relative to the tooling balls, the fibres were seen to return to the same position within $25 \mu \mathrm{m}$ during a test of about 100 actuations.

The greater part of the cantilever is made of a thin but rigid channel of $0.79 \mathrm{~mm}$ aluminum. To this were screwed two strips of $1.4 \mathrm{~mm}$ thick Macor [4] machinable ceramic, $41 \mathrm{~mm}$ long and $4.8 \mathrm{~mm}$ wide. The ends were ground with a radius of $4 \mathrm{~mm}$, centred near the inside edge. On the extreme edge, on the tangent between the two pieces, grooves for the three fibres were cut, spaced by $150 \mu \mathrm{m}$. With the fork held vertically, fibres were laid across the gap, and around the $4 \mathrm{~mm}$ radius of the Macor pieces, using a tension of $10 \%$ of their breaking strain. They were then fixed in place by immersion in a small spot of Sn96 solder, used without flux, on individual gold-plated copper tabs screwed to the Macor.

The cantilever design was tested and modified to ensure that its vibrational frequency was not close to a harmonic of the electrical power frequency. Microscope observations using the final design were carried out as close as possible to the actual operating position to ensure that transverse vibrations above about $1 \mu \mathrm{m}$ in amplitude were not occurring.

To allow flexibility in finding and measuring beams of a wide range of sizes, fibres of different diameters nominally 30,7 and $4.5 \mu \mathrm{m}$ - were installed on each holder. With the holder in the "in" position, the three fibres at its extreme tip were aligned perpendicular to the beam, and $150 \mu \mathrm{m}$ apart in a plane at $82^{\circ}$ to the beam axis.

Because of fears of charge-up and discharge damage near the beam, it is standard procedure to coat the surface of insulators, such as the Macor, with a conductor. In this case graphite has been used, but care was taken near the fibres to avoid shorting them electrically to ground or to each other.

Each terminal tab on the left side of the cantilever is connected to a $20 \mu \mathrm{m}$ gold-plated tungsten wire. This has been inserted in a sheath of fused silica tubing of outer diameter $150 \mu \mathrm{m}$, and shielded from radiofrequency pickup for most of its length by a stainless steel tube of diameter $0.36 \mathrm{~mm}$. This coaxial conductor takes the charge signal from the fibre along the cantilever, and connects to one of three miniature sockets in the web at the end. From there, each signal is passed around the circumference of the rocking ring to one of the vacuum feedthroughs. Wires with ceramic beads for insulation, and $0.94 \mathrm{~mm}$ diameter copper shielding jackets, were flexible enough to follow the circumference, and were used for this segment of the route. The overall design has the advantage that electrical connections in the vacuum are never flexed, even during actuation.

The target fibres are electrically connected to the outside world only at one end. By adjusting the thickness of the graphite coating around the other end of each fibre, it was possible to arrange that there would be a finite electrical resistance between the end of the fibre and the cantilever, which in turn is electrically coupled to the beam pipe. The resistance measured from the centre pin of a feedthrough to the beam pipe is the sum of this resistance plus the fibre resistance. It should be measurable if the fibre were intact, but would be effectively infinite if the fibre should break. This is used to check integrity during periods when the beam is absent. Some caution is warranted: the surface resistance of the graphite is susceptible to atmospheric moisture. The resistances we chose were in the $100 \mathrm{M} \Omega$ range, and in vacuo they became almost too high to measure.

All of the above materials and techniques were qualified for use in ultrahigh vacuum. The pipe was cleaned and baked during assembly, but the target cantilever and the connectors were not baked.

In the Mark II detector, the cantilever adds only about $7.5 \%$ to the scattering thickness of the beam pipe above $20^{\circ}$ from the beam axis. It also by itself is clear of small angle detectors inside $7^{\circ}$. There can, however, be as much as 1.7 radiation lengths of stainless steel, including structural material, flange, and the necessary mating flange, in front of parts of the end cap calorimeters.

\section{Control and signal acquisition}

The signals from the vacuum feedthroughs are connected through $10 \mathrm{~m}$ of RG223 cables to charge sensi- 
tive, ac coupled preamplifiers in a shielded environment. The preamplifiers and subsequent electronics are the same as those described in ref. [1]. In brief, the charge on a $1 \mathrm{nF}$ capacitance is altered by the change of charge in the fibre. A signal is generated which is proportional to this charge and peaks at $3 \mu$ s after beam crossing. This is transmitted to a remote 11-bit CAMAC ADC with a $0.5 \mu \mathrm{s}$ gate about the signal peak. The digitised amplitude is then accessed by a microcomputer in the SLC control and measurement network.

An alternative system is also in use to measure the shape of the beam at a fibre. The bremsstrahlung which occurs when beam particles traverse a fibre occupies essentially the same divergence cone as the charged beam, nominally about $200 \mu \mathrm{rad} \mathrm{rms}$. This radiation escapes the beam pipe through a thin window at $42 \mathrm{~m}$ from the collision point. There its intensity is measured by a Cherenkov counter immediately behind a 3 r.l. lead converter [5]. The data are accessed by the SLC computer similarly to the readout of the emitted charge.

Operation of the system starts by selecting the $X$ and/or $Y$ target for insertion on to the beam line. On request from one of the SLC control terminals, via one of the standard remote microcomputers, solenoid valves are actuated to shut off the pressure from, and vent, the retraction expansion chamber, and pressurise the insertion chamber. When the motion is completed, the electrical contacts indicate to the computer that the desired state has been reached. After this, a pattern of beam steering changes is selected, and pulse by pulse the beam is scanned across a fibre, while the fibre response is measured and read back, to be shown on a graphics display, plotted against the beam position.

\section{Utilisation at SLC}

The device has been used steadily when the final focus section of SLC has been under study, and more than a thousand beam scans have been made. It is used

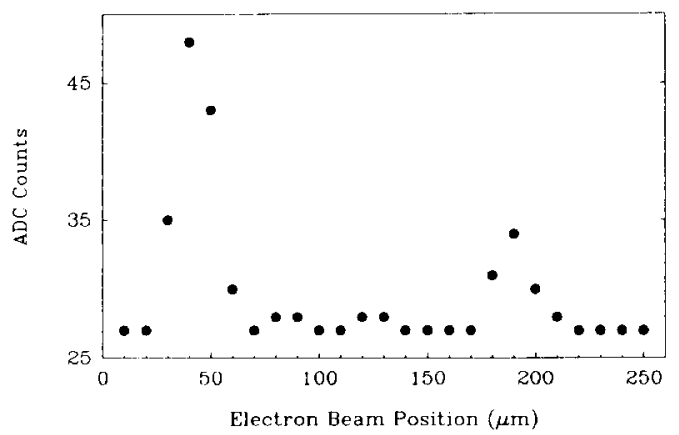

Fig. 3. Profiles of the beam as seen by 7 and $4.5 \mu \mathrm{m}$ fibres during a single scan, using the bremsstrahlung signal.

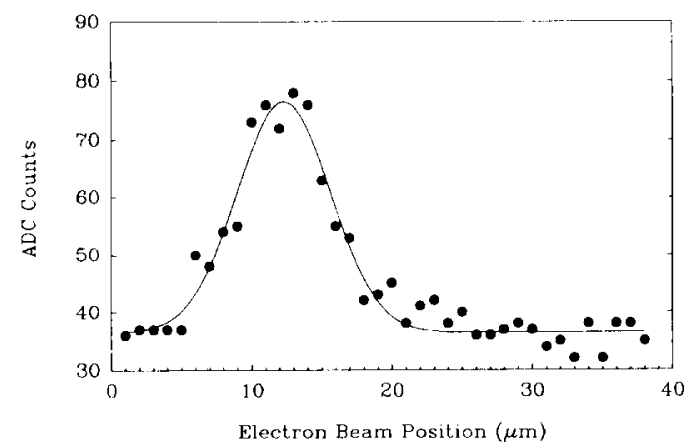

Fig. 4. A bremsstrahlung profile taken using a $4.5 \mu \mathrm{m}$ fibre. The indicated beam width is $3.3 \pm 0.17 \mu \mathrm{m}$ rms.

regularly to measure and to assist in minimising the beam size, and to find the waist and measure the angular divergence from a series of measurements as the focus is displaced along the beam line. It is also used to position the two beams so that they can be steered into collision. In carrying out these programs, the two measurement techniques have exhibited different characteristics, which we now review.

\section{Bremsstrahlung data}

Results from the bremsstrahlung detectors are illustrated in fig. 3, 4 and 5. The first shows a scan which traversed both 7 and $4.5 \mu \mathrm{m}$ fibres. Since the scan indicates that the space between the fibres is $150 \mu \mathrm{m}-$ the correct value - it is evident that the beam guiding system is calibrated accurately. The next illustration, fig. 4 , shows a scan across a $4.5 \mu \mathrm{m}$ fibre, with a width of $3.3 \pm 0.17 \mu \mathrm{m}$ from a Gaussian fit. In fig. 5 is shown a scan with a $7 \mu \mathrm{m}$ fibre, indicating a beam spot width of $3.22 \pm 0.08 \mu \mathrm{m}$.

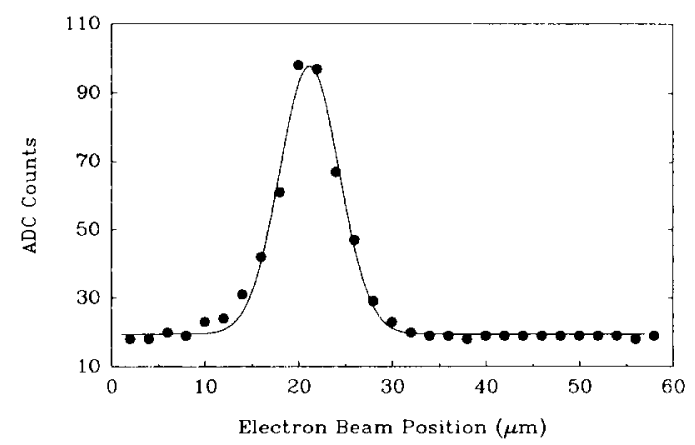

Fig. 5. A bremsstrahlung profile taken using a $7 \mu \mathrm{m}$ fibre. The rms width indicated is $3.22 \pm 0.08 \mu \mathrm{m}$, but after deconvoluting the size of the fibre, the beam width is calculated to be $2.7 \pm 0.14 \mu \mathrm{m}$. 
The fitted width is an overlap of the effects of the fibre shape and the beam width. Since bremsstrahlung is uniform in depth, the effect of the fibre can be represented simply by its rms thickness, which is (radius/2). Subtraction in quadrature for the example of fig. 5 leads to a true beam width of $2.7 \pm 0.14 \mu \mathrm{m}$ rms in the plane of the scan.

The uncertainty in the estimation of the beam width comes not only from the Gaussian fit to the observed peak, but also from the uncertainty in the width of the fibre where the beam hits it. The fibre width is not presently known to better than $\pm 0.7 \mu \mathrm{m}$, and, in the last example, its contribution to the unfolded width is $\pm 0.11 \mu \mathrm{m}$, compared with \pm 0.093 from the measurement of the peak. For beams narrower than this case, the $4.5 \mu \mathrm{m}$ fibres become valuable, despite their lower bremsstrahlung flux. Although their widths are not known better than those of the $7 \mu \mathrm{m}$ fibres, they would allow measurements of beams a factor of 0.75 as narrow as would the $7 \mu \mathrm{m}$ fibres, at the same uncertainty level.

Better measurements of the fibre thicknesses are being undertaken before the future scheduled beam operations. Nonetheless, the thinnest fibres will continue to provide the best estimates of the beam dimensions, and will be particularly important for widths much below $3 \mu \mathrm{m}$.

\section{Charge emission data}

Beam profiles can also be obtained by recording the charge emitted, pulse by pulse, as the beam is scanned across a fibre in the usual way [6]. This technique has been found to be less useful than bremsstrahlung measurement for several reasons.

In the case of positrons, the principal mechanism of surface emission is secondary emission. Since this occurs from a surface layer perhaps $50 \mathrm{~nm}$ thick, and is directly related to the energy-loss ionisation in this layer, the emission depends on $1 / \cos \Theta$, where $\Theta$ is the angle of the primary trajectory to the normal to the surface. Thus the response of a fibre to a scan of a Gaussian beam is not a simple function. An example is shown in fig. 6a. This may be compared with fig. 6b, which is for the same fibre and a beam of comparable size, but for an electron beam.

The very high radial electric fields in the neighbourhood of the short, dense SLC bunches mean that fields normal to the surface of an electrically conductive fibre immersed in a bunch are generally quite large. We estimate that, in a typical case with an electron beam, the induced charge on the fibre leads to a potential of several kilovolts to retard the emission of electrons. With a positron beam, on the other hand, the surface dipole field is reversed, and secondary electrons are extracted efficiently from the surface, despite any space
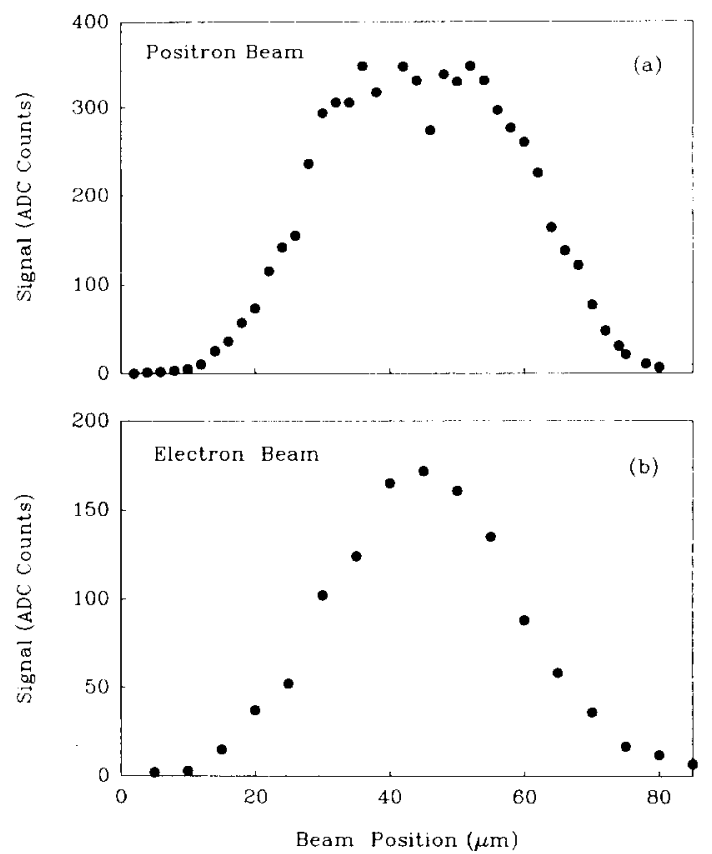

Fig. 6. (a) A profile taken with a narrow positron beam across a fibre of nominal diameter $30 \mu \mathrm{m}$, using the secondary emission signal. (b) A charge emission profile taken with an electron beam of comparable width and the same fibre. The beam intensities were approximately $2 \times 10^{9}$ and $6 \times 10^{9}$ respectively.

charge limit they would otherwise have. But in the case of electrons the true secondary emission is very heavily suppressed. The remaining emission is from delta rays with energies above the retarding potential at their point of emergence from the fibre. Thus the emission signal from the electron beam is an order of magnitude lower than from positrons. The effective shape of a fibre for the delta ray signal is quite different from the secondary emission response, and this is seen in the difference in the shapes in figs. $6 \mathrm{a}$ and $6 \mathrm{~b}$.

For the electron beam, the low yield has meant that the surface emission technique has not worked with the thinnest fibres. In addition, since it is considerably easier to extract the beam widths from the bremsstrahlung profile, the emission technique has less to offer. Further, it has been found that the signal becomes unstable for bunch populations above about $6 \times 10^{9}$, with large fluctuations in pulse amplitude. This may be caused by an instrumental failure to suppress effects of the radio-frequency impulse propagating in the beam pipe, or possibly by a physical effect of which we are not presently aware. It is known that reducing the signal level, and presumably noise level, in half by doubling the integrating capacitance before the preamplifier, does not substantially improve the breakup of the profiles. 
The bremsstrahlung technique has, however, been relatively trouble free, and so time has not been made available on the highly impacted accelerator schedule to study this problem.

\section{Discussion of some limitations}

The fibres are, of course, fragile, and require care in handling. There have been two instances of breakage during a year in the beam pipe. In both cases, $4.5 \mu \mathrm{m}$ fibres failed. The causes are not known, although they are not believed to be associated with the beam damage described below. The other fibres have served well and have so far imposed no operational limitations or lost time.

In addition to the limited range of the emission readout, there is a fundamental limit on the use of the fibres in intense narrow beams. Most of the ionisation energy loss is available to heat the fibre, although we estimate that $37 \%$ of the ionisation energy escapes as delta rays from a $30 \mu \mathrm{m}$ fibre $(41 \%$ and $45 \%$ escape from 7 and $4 \mu \mathrm{m}$ fibres respectively). Estimation of the temperature which the fibre reaches is hampered by the inadequate information available about the thermal properties of carbon fibres at elevated temperatures. The following numbers are therefore rather uncertain, but serve as a guide for operational limitations.

As an example, a beam of $2 \times 10^{10}$ particles, with a $5 \mu \mathrm{m}$ Gaussian radius, should raise the temperature where its centre penetrates a fibre by about $1580^{\circ} \mathrm{C}$. The small hot spot would cool by conduction in some tens of microseconds, well before the next pulse would arrive. However, we calculate that, for temperatures above about $2200^{\circ} \mathrm{C}$, the shock wave accompanying the thermal impulse would exceed the elastic limits of the material, and failure may result. The limit is yet to be tested. However, the tuning of beams with densities somewhere above $2 \times 10^{10}$ and $5 \mu \mathrm{m}$ Gaussian radius will require other techniques than fibre targets.

\section{Summary}

The fibre targets, with a bremsstrahlung readout, have operated successfully for a year inside the Mark II detector. There has been little interference with the operation of the detector, except, of course, when beam tuning is in progress. The surface emission signal from the fibres has been found to have systematic difficulties which limit its use for short bunches with bright beam spots. Fibre targets have a limited range of application because of heating effects, but this limit has not yet been reached.

\section{Acknowledgements}

The help and cooperation of the Vacuum Group, particularly M. Hoyt, is greatly appreciated, as is the help of V. Price of the Instrumentation and Control Group. Dr. N. Phinney and A. Gromme contributed substantially to the programming of the SLC control computer to implement the beam scanning function. Dr. R. Frey was responsible for the microcomputer code which reads out the bremsstrahlung signal. We were fortunate to be able to leave the delicate task of handling the carbon fibres in the skilful hands of Mrs. Y.Y. Sung.

\section{References}

[1] R. Fulton et al., Nucl. Instr. and Meth. A274 (1989) 37.

[2] G. Abrams et al., SLAC-PUB-4558 (Dec. 1988) submitted to Nucl. Instr. and Meth.

[3] SLC Design Report, SLAC (Dec. 1984) p. 4-24; R. Erickson, SLAC Memorandum: Dither Coils for the Final Focus (June 3, 1986).

[4] Corning Glass Works, Corning, New York, USA.

[5] G. Bonvicini et al., SLAC-PUB-4735 (October, 1988) to be published in Nucl. Instr. and Meth.

[6] A more detailed discussion of this topic than can be given here is in preparation. 